\title{
Linfedema escrotal masivo: Reporte de caso y revisión de la literatura
}

\author{
Massive scrotal lymphedema: case report and literature review
}

\author{
Melquíades Álvarez-Chaires ${ }^{1}$, Rogelio Martínez-Wagner², Iván Ortiz-Monasterio Todd ${ }^{3}$, \\ Mario A. Morán-Romero ${ }^{3}$, Nicolás Hernández-García ${ }^{4}$, José M. Álvarez Diaz ${ }^{1}$
}

\begin{abstract}
RESUMEN
El lindefema masivo localizado es una condición rara pero su incidencia se encuentra en aumento. El linfedema puede ser primario o secundario a infecciones, radioterapia, cirugías, cáncer, enfermedades del sistema inmune o a compresión. Algunos autores han sugerido un incremento en su incidencia debido a la epidemia de obesidad. El linfedema escrotal produce una morbilidad importante debido a mala higiene, infecciones, incontinencia urinaria, disfunción sexual, pérdida de la libido e inclusive limitación en la deambulación. Las opciones de tratamiento no quirúrgico de la linfedema incluyen el drenaje linfático manual, prendas de compresión, terapia física y ejercicio. Usualmente, el tratamiento conservador falla debido a la dificultad anatómica que presenta la región genital para la aplicación de dichas terapias. El tratamiento quirúrgico puede ser efectivo en casos graves y moderados. Existen diferentes variantes a la técnica quirúrgica, pero la resección del tejido afectado y la reconstrucción con tejido sano es primordial. El pronóstico de esta patología tiende a ser bueno; sin embargo, se reporta una alta tasa de recidiva.
\end{abstract}

Palabras claves: linfedema escrotal masivo, linfedema masivo, linfedema escrotal, tumor escrotal.

\begin{abstract}
Massive localized lymphedema is a rare but rising condition that could be primary in nature or secondary to infections, radiation, surgery, malignancies, autoimmune or compression. Some authors have suggested and increased incidence due to the raising epidemic of obesity. Scrotal lymphedema causes significant morbidity such as poor hygiene, infections, urinary incontinence, sexual dysfunction, loss of libido and a limited ambulation. Although non operative treatment options for lymphedema include manual lymph drainage, compression garments, exercises, sequential gradient pump. Usually, non-operative techniques fail due to the difficult in the anatomic area. Surgical treatment can be effective in moderate to severe cases of giant scrotal lymphedema. There are many variations of the surgical technique, however, resection of the affected tissue and reconstruction with healthy skin is primordial. The prognosis tends to be good, although there is reported a significant amount of relapse, mostly dependent on the weight lost and diet of the patients.
\end{abstract}

Key words: massive scrotal lymphedema, massive lymphedema, scrotal lymphedema, scrotal tumor.

REVISTA ARGENTINA DE CIRUGÍA PLÁSTICA 2020;26(2):88-91. HTTPS://DOI.ORG/10.32825/RACP/202002/0088-0091

\section{INTRODUCCIÓN}

El linfedema masivo localizado es una entidad rara y debilitante, aunque su incidencia parece estar en aumento. Se caracteriza por la obstrucción del drenaje linfático de una región del cuerpo que genera un aumento de volumen desproporcionado.

Las causas de esta condición clínica pueden ser divididas en primarias, causas congénitas primordialmente o secundarias. Las causas secundarias alteran el flujo de linfa y obedecen a diferentes etiologías: obstruc-

1. Médico de base. Departamento de Cirugía Plástica y Reconstructiva, Hospital Guadalupe, Matamoros, Tamaulipas, México.

2. Médico de base. División de Cirugía Plástica y Reconstructiva, Hospital General "Dr. Manuel Gea González" Ciudad de México, México.

3. Residente. División de Cirugía Plástica y Reconstructiva, Hospital General "Dr. Manuel Gea González" Ciudad de México, México.

4. Médico de base. Departamento de Urología, Hospital Guadalupe, Matamoros, Tamaulipas, México.

$\square$ Correspondencia: Mario Arturo Morán-Romero. División de Cirugía Plástica y Reconstructiva, Hospital General "Dr. Manuel Gea González" Ciudad de México, México. Calz. de Tlalpan 4800, Belisario Domínguez Secc 16, 14080 Ciudad de México, CDMX. Email: marioarturom@gmail.com ción de linfáticos por parásitos (Wuchereria bancrofti), radioterapia, cirugías, tumores, cáncer, enfermedades autoinmunes, compresión de conductos linfáticos, casos idiopáticos y obesidad ${ }^{1-3}$.

El linfedema escrotal masivo (LEM), en ocasiones llamado elefantiasis penescrotal, tiene una epidemiología desconocida debido a su baja frecuencia y a la falta de consenso respecto a su definición y manejo ópti$\mathrm{mo}^{4}$. En este trabajo, reportamos el caso de un paciente con LEM tratado de forma satisfactoria mediante resección en bloque y reconstrucción inmediata con colgajos de piel escrotal sana.

\section{REPORTE DE CASO}

Se trata de un paciente masculino de 27 años, con un peso de $153 \mathrm{~kg}$ (IMC 39.4), quien presenta crecimiento localizado en el escroto de 18 meses de evolución. El paciente tenía antecedente de una torsión testicular derecha que requirió tratamiento quirúrgico no especificado cuando tenía 2 años. El paciente no reportaba dolor, pero el tumor causaba alteraciones significativas en su calidad de vida. El paciente era incapaz de realizar actividades de la vida cotidiana, además era incapaz de mantener relaciones sexuales. Al examen físico presentaba aumento de volumen escrotal difuso 
TABLA 1. Técnicas de tratamiento quirúrgico y complicaciones para el tratamiento del linfedema escrotal masivo (LEM).

\begin{tabular}{|c|c|c|c|c|c|c|}
\hline Autores & $\begin{array}{l}\text { Año de } \\
\text { publicación }\end{array}$ & $\begin{array}{l}N^{\circ} \text { de } \\
\text { casos }\end{array}$ & $\begin{array}{l}\text { Preservación } \\
\text { testicular }\end{array}$ & $\begin{array}{l}\text { Preservación } \\
\text { de cordón } \\
\text { espermático }\end{array}$ & Procedimiento reconstructivo & Complicaciones \\
\hline Dandapat MC $\mathrm{C}^{11}$ & 1985 & 350 & $\mathrm{SI}$ & 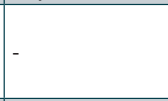 & IEPP & $\begin{array}{l}\text { Hematoma }(8.2 \%) \text {, Absceso/ } \\
\text { infección (10.6\%), recurren- } \\
\text { cia }(7.13 \%) \text {. }\end{array}$ \\
\hline Kuepper D ${ }^{12}$ & 2005 & 1 & No & No & $\begin{array}{l}\text { Periné cierre primario. Diáfisis de } \\
\text { pene cierre por } 2 \text { da intención e IEPP }\end{array}$ & Ninguna. \\
\hline Hornberger $\mathrm{B}^{13}$ & 2005 & 1 & $\mathrm{Si}$ & - & $\begin{array}{l}\text { Escroto: colgajos cutáneos basados } \\
\text { en piel redundante de área suprapú- } \\
\text { bica. Pene: preservación de piel. }\end{array}$ & $\begin{array}{l}\text { Pérdida de seguimiento, ede- } \\
\text { ma. }\end{array}$ \\
\hline Denzinger $S^{14}$ & 2007 & 1 & $\mathrm{Si}$ & Si & $\begin{array}{l}\text { Escroto: colgajos de rotación. Pene: } \\
\text { preservación de piel. }\end{array}$ & $\begin{array}{l}\text { Desbridamientos seriados, te- } \\
\text { rapia VAC e IEP para recons- } \\
\text { trucción definitiva. } \\
\end{array}$ \\
\hline Zacharakis $\mathrm{E}^{15}$ & 2008 & 1 & $\mathrm{Si}$ & $\mathrm{Si}$ & $\begin{array}{l}\text { Escroto y pene, colgajos de piel es- } \\
\text { crotal. }\end{array}$ & Ninguna. \\
\hline Rahaman $\mathrm{G}^{16}$ & 2009 & 1 & $\mathrm{Si}$ & NE & $\begin{array}{l}\text { Escroto: cierre por } 2 \text { da intención. } \\
\text { Pene: IEP. }\end{array}$ & Ninguna. \\
\hline Dianzani $C^{17}$ & 2010 & 1 & $\mathrm{Si}$ & Si & $\begin{array}{l}\text { Escroto: colgajos fasciocutáneo de } \\
\text { avance en VY. Pene: IET. }\end{array}$ & Ninguna. \\
\hline Hidalgo $\mathrm{ER}^{18}$ & 2010 & 1 & $\mathrm{Si}$ & $\mathrm{Si}$ & Pene y escroto: IEP & Ninguna. \\
\hline Talikoti $\mathrm{M}^{19^{*}}$ & 2011 & 1 & No & - & $\begin{array}{l}\text { Escroto: colgajo de rotación fasciocu- } \\
\text { táneo suprapúbico. }\end{array}$ & Ninguna. \\
\hline Thejeswi P20 & 2012 & 1 & No & No & $\begin{array}{l}\text { Escroto: cierre en Y. Pene: preserva- } \\
\text { ción de piel. }\end{array}$ & Ninguna. \\
\hline Champaneria $\mathrm{M}^{4}$ & 2013 & 1 & $\mathrm{Si}$ & $\mathrm{Si}$ & $\begin{array}{l}\text { Escroto: Colgajo fasciocutáneo pos- } \\
\text { terior (Fascia dartos). Pene: Injerto de } \\
\text { piel y colgajo fasciocutáneo local. }\end{array}$ & Ninguna. \\
\hline Parmar $\mathrm{H}^{21}$ & 2013 & 1 & $\mathrm{Si}$ & NE & $\begin{array}{l}\text { Escrotoplastia con sutura en línea } \\
\text { media. Pene: IEP. }\end{array}$ & Ninguna. \\
\hline Brotherhood $\mathrm{HL}^{7}$ & 2014 & 2 & $\mathrm{Si}$ & $\mathrm{Si}$ & $\begin{array}{l}\text { Paciente 1: Escroto, colgajo escrotal. } \\
\text { Pene, IEP. Paciente 2: Escroto, colga- } \\
\text { jo escrotal. Pene, avance de colga- } \\
\text { jo e IEP. }\end{array}$ & $\begin{array}{l}\text { Paciente 1: Ninguna. } \\
\text { Paciente 2: Infección sitio qui- } \\
\text { rúrgico, epidermólisis. }\end{array}$ \\
\hline Machol JA 22 & 2014 & 4 & $\mathrm{Si}$ & No & $\begin{array}{l}\text { Colgajo escrotal, IEP, Z plastia, Colga- } \\
\text { jos en VY. }\end{array}$ & $\begin{array}{l}\text { Dehiscencia }(75 \%) \text {, infección } \\
(50 \%) \text {, recurrencia }(50 \%) \text {. }\end{array}$ \\
\hline Ravari H²3 & 2015 & 1 & $\mathrm{Si}$ & Si & Cierre en forma de Y. & Ninguna \\
\hline Qing Lu & 2016 & 17 & $\mathrm{Si}$ & Si & $\begin{array}{l}\text { Anastomosis linfático venosa (hiper- } \\
\text { plasia y disfunción nodal moderada) } \\
\text { Técnica excisional (Disfunción nodal } \\
\text { moderada y severa) ( } n=17 \text { ) }\end{array}$ & $\begin{array}{l}\text { Hematoma }(5.9 \%), \text { Sin re- } \\
\text { currencia a } 5 \text { años de segui- } \\
\text { miento. }\end{array}$ \\
\hline Salako A ${ }^{25}$ & 2016 & $\begin{array}{l}11 \mathrm{Qx} \\
19 \mathrm{Tx}\end{array}$ & $\mathrm{Si}$ & Si & Colgajos escrotales. & $\begin{array}{l}\text { Hematoma }(27.4 \%) \text {, infección } \\
(18.2 \%) \text {, recurrencia }(9.1 \%) \text {. }\end{array}$ \\
\hline Wisenbaugh $\mathrm{E}^{26}$ & 2016 & 11 & $\mathrm{Si}$ & Si & Colgajos de piel, injertos de piel ( $n=1)$ & $\begin{array}{l}\text { Dehiscencia (54.5\%), infec- } \\
\text { ción }(9 \%) \text {, edema recurrente } \\
(9 \%) \text {, lesión de plexo braquial } \\
(9 \%) \text {, insuficiencia respirato- } \\
\text { ria }(9 \%) \text {. }\end{array}$ \\
\hline Vives $F^{5}$ & 2016 & 1 & $\mathrm{Si}$ & Si & $\begin{array}{l}\text { Procedimiento en } 4 \text { etapas } \\
\text { Colgajo de piel escrotal sana. }\end{array}$ & Ninguna. \\
\hline Salako $A^{27}$ & 2018 & 11 & - & - & Colgajo de piel escrotal sana. & Ninguna. \\
\hline Pacheco Y28 & 2018 & 1 & $\mathrm{Si}$ & $\mathrm{Si}$ & $\begin{array}{l}\text { Resección y cobertura retrasada, IEP } \\
\text { diáfisis de pene, IET testículos. }\end{array}$ & $\begin{array}{l}\text { Pérdida del injerto en diáfisis } \\
\text { de pene. }\end{array}$ \\
\hline Hori $\mathrm{S}^{29 * *}$ & 2018 & 1 & Si & $\mathrm{Si}$ & Colgajo de la piel del pene. & Ninguna. \\
\hline Elkiran Y30 & 2019 & 1 & $\mathrm{Si}$ & $\mathrm{Si}$ & $\begin{array}{l}\text { Escrotoplastia con preservación de } \\
\text { piel sana del pene. }\end{array}$ & Dehiscencia y necrosis de piel. \\
\hline
\end{tabular}

con piel de naranja y cambios de coloración, hiperpigmentación especialmente en el área cercana a los muslos. A la palpación no presentaba dolor, pero sí aumento en la consistencia. El pene no era visible y los testículos no podían ser palpados (Figura 1). Sus estudios de laboratorio no demostraron alteraciones. El ultrasonido reportó ambos testículos con un tamaño y ecogenicidad normal, rodeados de abundante tejido fibrograso. La tomografía computarizada demostraba la ocupación completa del escroto sin presencia de calcificaciones ni infiltrados.
Bajo anestesia general se realizó el procedimiento quirúrgico. La incisión en piel se realizó sobre el rafe, en línea media. Se realizó una resección en bloque del tejido afectado, se desenterró el pene, de forma inicial se localizaron y preservaron los testículos y cordones espermáticos (Figura 2). La reconstrucción del escroto y la cobertura del pene se realizó con colgajos fasciocutáneos bilaterales. Se colocó un drenaje cerrado y se realizó el cierre de colgajos en línea media (Figura 3). El paciente se recuperó sin presentar complicaciones. El estudio histopatológico fue negativo para malignidad. 


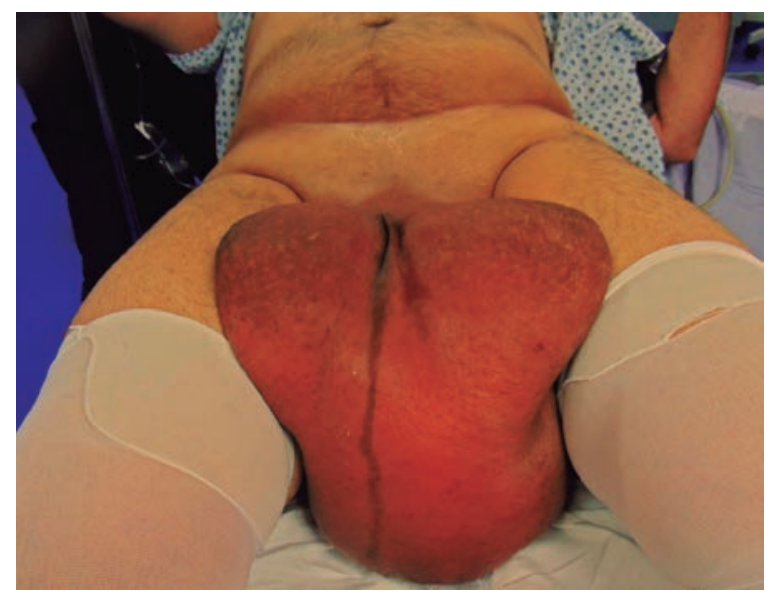

Figura 1. Marcaje preoperatorio, se dibuja una incisión en la línea media a lo largo del rafe escrotal.

\section{DISCUSIÓN}

El LEM es poco frecuente, tiene una naturaleza crónica y progresiva debido a la disfunción del sistema linfático. En los países tropicales el linfedema escrotal es generalmente causado por linfogranuloma venéreo o filariasis $^{5}$. La enfermedad produce una morbilidad significativa en el paciente debido a mala higiene, incontinencia urinaria, disfunción sexual, pérdida de la libido y limitación a la deambulación.

El diagnóstico es clínico, aunque diferentes estudios han ganado popularidad debido al incremento en la frecuencia de la patología. Algunas de las pruebas más utilizadas son la linfocentellografía, la linfografía directa y la indirecta. Asimismo, la resonancia magnética, la tomografía computarizada y el ultrasonido permiten demostrar edema limitado a los compartimentos superficiales con un patrón reticular difuso e hipertrofia de la piel, los compartimentos musculares se encuentran respetados. Dependiendo del tiempo de evolución, de forma inicial se observa un estado de inflamación crónica que condiciona engrosamiento y aumento en la consistencia de la piel y el tejido subcutáneo, lo cual produce una sensación de pesadez en la región afectada. En algunas ocasiones, cierto flujo de linfa puede ser visto a través de la piel y ocurre la impetiginización. ${ }^{4}$

Aunque las opciones de tratamiento no quirúrgico de linfedema incluyen las prendas de compresión, ejercicios y bombeo de gradiente secuencial ${ }^{6}$, usualmente el tratamiento conservador falla debido a la complejidad del área anatómica.

La resección quirúrgica puede ser un tratamiento efectivo en casos de LEM, Sin embargo, el tratamiento quirúrgico requiere mantener el potencial reproductivo y mejorar la función sexual, especialmente en el caso de pacientes jóvenes. Los procedimientos microquirúrgicos incluyen la linfangioplastia o anastomosis linfático-venosas; la aplicación de dichos tratamientos está limitada a pacientes en etapas iniciales.

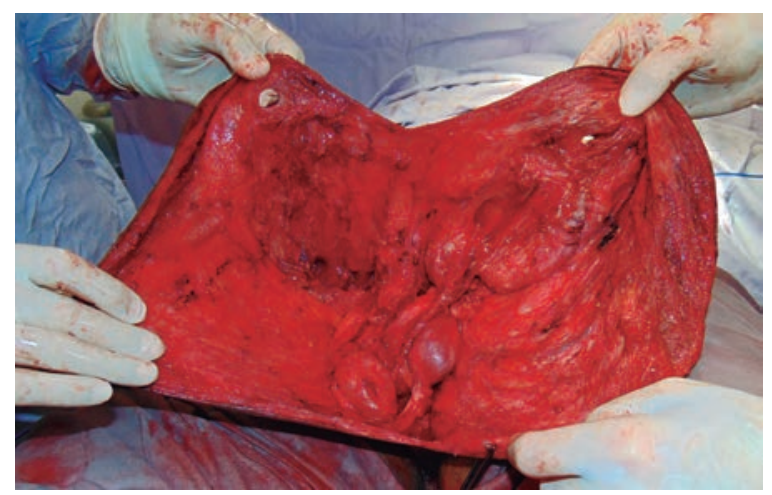

Figura 2. Fotografía intraoperatoria mostrando la disección de colgajos de piel, se muestra la preservación de los testículos y cordones espermáticos.

Cuando la enfermedad es extensa, la resección quirúrgica del tejido afectado es el tratamiento preferido. La preservación de los cordones espermáticos y otras estructuras genitourinarias es de primordial importancia. Pocos estudios reportan el resultado de la función sexual en este grupo específico de pacientes. Nuestro paciente reportó mejoría de la función sexual posterior al procedimiento, aunque no se realizó ninguna prueba específica para evaluar este aspecto. Nosotros recomendamos realizar una evaluación multidimensional en los pacientes que son afligidos por esta condición clínica.

Algunos autores proponen la realización de varios procedimientos para tratar de forma exitosa esta condición ${ }^{5}$. En 1820, Delpech describió el tratamiento quirúrgico de la elefantiasis genital mediante la resección de toda la piel u tejido subcutáneo con linfedema, la reconstrucción se realizó mediante la aplicación de injertos de espesor parcial en el pene y el escroto lo reconstruyó mediante el avance de colgajos de piel sana del periné posterior ${ }^{7}$. Existen comunicaciones en la literatura de diferentes opciones de reconstrucción que consisten en el uso de colgajos locorregionales, injertos de piel, combinaciones de colgajos e injertos e incluso el cierre asistido por vacío (Tabla $\mathbf{1}$ ).

Es difícil establecer el pronóstico en esta patología debido a su baja frecuencia y muchos de los autores describen una evolución posoperatoria favorable durante el seguimiento del paciente. En algunas series se reporta hasta una recurrencia del $50 \%$ de los $\operatorname{casos}^{8}$. La infección del sitio quirúrgico y la dehiscencia son complicaciones frecuentes del procedimiento. El diseño adecuado del colgajo de piel escrotal sana es clave para asegurar la viabilidad del tejido y prevenir la recurrencia. Los colgajos escrotales brindan ventaja sobre los injertos pues mantienen la termorregulación. Los injertos de espesor parcial (IEP) son una excelente opción para la cobertura del escroto cuando no se encuentra piel escrotal sana. Los IEP ofrecen una apariencia más natural, temperatura menor a la abdominal, morbilidad 


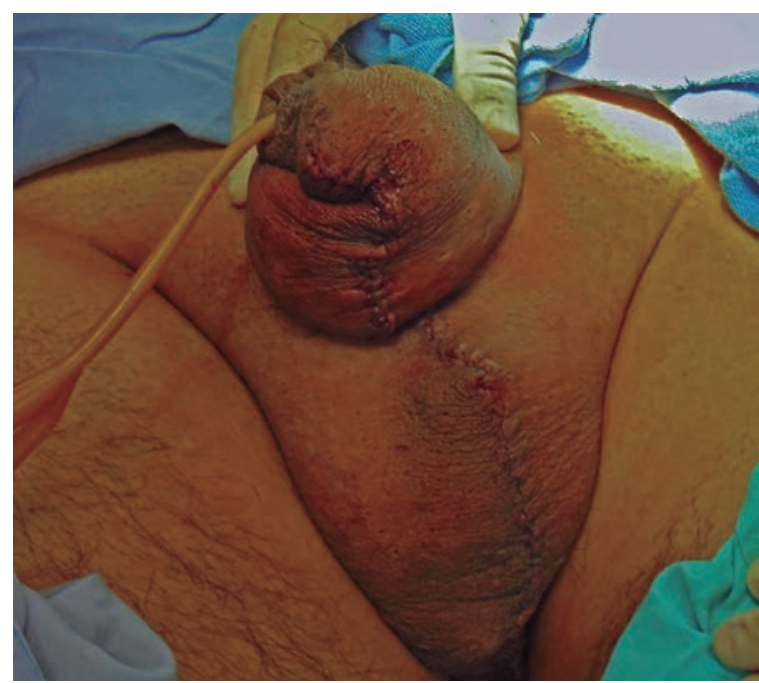

Figura 3. Resultado posoperatorio inmediato mostrando la reconstrucción escrotal con colgajos de piel. mínima y generalmente se pueden realizar en el mismo tiempo quirúrgico. El cierre asistido por vacío (VAC, siglas en inglés) es una excelente opción para el manejo de la dehiscencia o infecciones. En dichos casos la cobertura puede ser lograda mediante la realización tardía de un IEP.

\section{CONCLUSIONES}

El LEM es una condición que produce alteraciones físicas y psicológicas. Actualmente su presentación es baja pero se encuentra en aumento. El incremento se encuentra relacionado con la obesidad. El tratamiento incluye el tratamiento quirúrgico y el conservador. En las etapas tempranas se recomienda realizar la reconstrucción microquirúrgica. La resección y posterior reconstrucción se recomienda en las etapas avanzadas de la enfermedad.

\section{BIBLIOGRAFÍA}

1. McDougal WS. Lymphedema of the external genitalia. J Urol 2003 Sep;170(3):711-6.

2. Szuba A, Rockson SG. Lymphedema: classification, diagnosis and the rapy. Vasc Med 1998:3(2):145- 56

3. Garfein ES, Borud LJ, Warren AG, et al. Learning from a lymphedema clinic: an algorithm for the management of localized swelling. Plast Reconstr Surg 2008;121(2):521-8.

4. Champaneria MC, Workman A, Kao H, et al. Reconstruction of massive localised lymphoedema of the scrotum with a novel fasciocutaneous flap: A rare case presentation and a review of the literature. $J$ Plast Reconstr Aesthet Surg 2013;66(2):281-6.

5. Vives F, García-Perdomo HA, Ocampo-Flórez GM. Giant lymphedema of the penis and scrotum: a case report. Autops Case Rep 2016;6(1):57-61.

6. Schaverien MV, Moeeler JA, Cleveland SD. Nonoperative treatment of lymphedema. Semin Plast Surg 2018;32(1):17-21.

7. Brotherhood HL, Metcalfe M, Goldenberg L, et al. A surgical challenge: Idiopathic scrotal elephantiasis. Can Urol Assoc J 2014;8(78):E500-7.

8. Farshid G, Weiss SW. Massive localized lymphedema in the morbidly obese: a histologically distinct reactive lesion simulating liposarcoma. Am J Surg Pathol 1998;22(10):1277-83.

9. Tosun Z, Sutcu M, Guven S, et al. Giant scrotal elephantiasis with unknown etiology. Ann Plast Surg 2005;54(6):685-6.

10. Lucas JW, Lester KW, Chen A, et al. Scrotal reconstruction and testicular prosthetics. Transl Androl Urol 2017;6(4):710-21.

11. Dandapat MC. Elephantiasis of the penis and scrotum. A review of 350 cases. Am J Surg 1985;149(5):686-90.

12. Kuepper D. Giant scrotal elephantiasis. Urology 2005;65(2):389.

13. Hornberger BJ, Elmore JM, Roehrborn CG. Idiopathic scrotal elephantiasis. Urology 2005;65(2):389.

14. Denzinger $S$, Watzlawek E, Burger M. Giant scrotal elephantiasis of inflammatory etiology: a case report. J Med Case Rep 2007;2:1-23.

15. Zacharakis E, Dudderidge T, Zacharakis $E$, et al. Surgical repair of idiophatic scrotal elephantiasis. South Med J 2008:101(2):208-10.

16. Rahman GA, Ismaila AA, Ibrahim FY, et al. Giant scrotal lymphedema of unclear etiology: a case report. J Med Case Reports 2009;3:7295.
17. Dianzani C, Gaspardini F, Persichetti P, et al. Giant scrotal elephantiasis: an idiopathic case. Int J Immunopathol Pharmacol 2010;23(1):369-72.

18. Hidalgo ER, García-Moreno AL, González EB, et al. Surgical treatment in a case of giant scrotal lymphedema. Arch Esp Urol 2011;64(2):121-4.

19. Talikoti MA, Deo SV, ShuklaNk, et al. A rare case of giant leiomyosarcoma in a filarial scrotum: a case report. World Surg Oncol 2011;9:20.

20. Thejeswi P, Prabhu S, Augustine AJ, et al. Giant scrotal lymphoedema. A case report. Int J Surg Case Rep 2012,3(7):269-71.

21. Parmar HD. The surgical approach in huge scrotal lymphedema. Int J Med Sci Public Health 2013;2(1):153-5.

22. Machol JA, Langenstroer $P$, Sanger JR. Surgical reduction of scrotal massive localized lymphedema (MLL) in obesity. J Plast Reconstr Aesthet Surg 2014:67(12):1719-25.

23. Ravari H, Ghoddusi JH, Rajabnejad A, et al. Gian Scrotal lymphoedema. J Cutan Aesthet Surg 2015;8(1):67-8.

24. Qing L, Zhaohua J, Zizhou Z, et al. Assessment of the lymphatic system of the genitalia using magnetic resonance lymphography before and after treatment of male genital lymphedema. Medicine (Baltimore) 2016;95(21):e3755.

25. Salako $A A$, Olabanji $J K$, Oladele $A O$. Surgical reconstruction of giant penoscrotal lymphedema in Sub-Saharan Africa. Urology 2018:112:181-5.

26. Wisenbaugh E, MoskowitzD, Gelman J. Reconstruction of massive localized lymphedema of the scrotum: results, complications, and quality of life improvements. Urology 2018;112:176-80.

27. Salako AA, Olabanji JK, Oladele $A O$, et al. Surgical reconstruction fo giant penoscrotal lymphedema in Sub-Saharan Africa. Urology 2018;112:181-5.

28. Pacheco YD, García-Duque O, Fernández-Palacios J. Penile and scrotal lymphedema associated with hidradenitis suppurativa: Case report and review of surgical options. Cir Cir 2019;86(1):77-80.

29. HoriS, MitsuiY, IwaiH, et al. Giant penilelymphedema caused by chronic penile strangulation with rubber band: a case report and review of the literature. Case Rep Urol 2018; 2018:8598195.

30. Elkiran YM, Elshafer AM, Mohammed SW, et al. Surgical manage ment of giant scrotal lymphedema in morbidly obese patient with trisomy 21. J Vas Surg Cases Innov Tech 2019;5(1):71-4. 